\title{
Associations Between Mental Health, Quality of Life, and Obesity/Metabolic Risk Phenotypes
}

\author{
Se-Rae Kim, MD, Ha-Na Kim, MD, PhD, and Sang-Wook Song, MD, PhD
}

\begin{abstract}
Background: Obesity is a risk factor for many health issues, as are metabolic abnormalities; all may affect mental health and one's health-related quality of life. Therefore, we investigated the association between obesity subtypes, considering both body weight and metabolic abnormalities, and the mental health and quality of life of a Korean population.

Methods: We used data from the 2016 Korean National Health and Nutrition Examination Survey, a crosssectional survey of Korean civilians, and data on a total of 6057 participants were analyzed. Obesity subtypes were classified as metabolically healthy but obese (MHO); metabolically abnormal but of normal weight (MANW); and metabolically abnormal and obese (MAO).

Results: MHO was positively associated with mobility problems, pain/discomfort, and stress compared with metabolically healthy and of normal weight (MHNW) control. MAO was positively associated with problems in terms of not only mobility problems, pain/discomfort, and stress but also self-care and usual activity problems, and improper sleep duration, compared with the MHNW control. MANW showed no associations with the mental health problems or quality of life.

Conclusions: With or without metabolic abnormalities, obesity is associated with mental health problems and decreased quality of life.
\end{abstract}

Keywords: obesity, metabolic syndrome, mental health, quality of life

\section{Background}

$\mathrm{O}$ BESITY, WHICH IS DEFINED as excess accumulation of body fat, is of major global concern; the incidence is increasing. ${ }^{1}$ Obesity is associated with cardiometabolic disease, including hypertension, diabetes mellitus, dyslipidemia, and cardiovascular diseases, ${ }^{2}$ and obesity-induced insulin resistance and metabolic abnormalities also cause adverse cardiometabolic outcomes; all may affect mental health and one's health-related quality of life. ${ }^{3-6}$

However, not all obese individuals show metabolic abnormalities, and not all normal-weight individuals show favorable metabolic conditions. Therefore, obesity subtypes divided according to metabolic status such as metabolically healthy but obese (MHO), metabolically abnormal but of normal weight (MANW), and metabolically abnormal and obese (MAO) have attracted attention. ${ }^{7}$

Several studies have examined the association of obesity subtypes with quality of life and mental health ${ }^{8-11}$; however, studies investigating the associations between obesity subtypes and mental health or quality of life are scare in Asian populations. Therefore, we investigated whether various obesity subtypes are associated with mental health and quality of life using data from the Korean National Health and Nutrition Examination Survey (KNHANES).

\section{Materials and Methods}

\section{Study population}

We used data from the 2016 KNHANES. The KNHANES is conducted by the Korean Center for Disease Control and Prevention at 3-year intervals to assess public health status and provide baseline data facilitating the development, establishment, and evaluation of public health policies. The KNHANES subjects are noninstitutionalized and aged $\geq 1$ year; they are selected using a stratified, multistage, cluster probability sampling design to ensure that the sample is

Department of Family Medicine, St. Vincent's Hospital, College of Medicine, The Catholic University of Korea, Seoul, Republic of Korea. 
independent, homogeneous, and nationally representative. Data are collected using household interviews and via anthropometric and biochemical measurements, and include nutritional status assessments. All protocols have been approved by the Institutional Review Board of the Korean Center for Disease Control and Prevention, and all participants provided written informed consent at baseline.

In this cross-sectional study, we originally examined 6315 adults $\geq 20$ years of age selected from 8150 KNHANES participants. We excluded those for whom important data were missing $(n=12)$ and those with histories of psychiatric diseases $(n=246)$. Thus, we finally evaluated 6057 subjects (Fig. 1).

The study was approved by the Institutional Review Board of the Catholic University of Korea (IRB approval no.: VC18ZESI0158).

\section{Classification of obesity subtypes}

Participants were classified as being of normal weight or obese using body mass index (BMI) of $<25$ and $\geq 25.0 \mathrm{~kg} / \mathrm{m}^{2}$, respectively. ${ }^{12}$ Metabolically abnormal status was defined as the presence of any three or more of the following (consistent with the revised National Cholesterol Education Program Adult Treatment Panel III definitions of metabolic syndrome $[\mathrm{MetS}])^{13}$ : waist circumference $\geq 90 \mathrm{~cm}$ for males $(\geq 85 \mathrm{~cm}$ for females) ${ }^{14}$; triglyceride level $\geq 150 \mathrm{mg} / \mathrm{dL}$ or on medication to reduce triglyceride levels; high-density lipoprotein cholesterol (HDL-C) level $<40 \mathrm{mg} / \mathrm{dL}$ for males $(<0 \mathrm{mg} / \mathrm{dL}$ for females) or on medication to increase HDL-C levels; systolic blood pressure $\geq 130 \mathrm{mmHg}$ or diastolic blood pressure $\geq 85 \mathrm{mmHg}$ or on an antihypertensive agent; and a fasting glucose level $\geq 100 \mathrm{mg} / \mathrm{dL}$ or on a blood glucose-lowering agent. All subjects were divided into four groups based on the presence/absence of obesity and MetS: metabolically healthy and of normal weight (MHNW) control, MHO, MANW, and MAO.

\section{Definitions of variables}

We evaluated four psychiatric symptoms. Sleep time was classified into two categories: proper ( $\geq 7 \mathrm{hr}$ ) and improper $(<7 \mathrm{hr})$. All subjects were asked to self-report whether they experienced stress in their daily lives, and responded "yes" or "no." Depressed mood was defined as an affirmative response to "Have you ever felt so sad or hopeless that you experienced difficulties in daily life on a continuous basis for more than 2 weeks during the past year?" We asked subjects if they had ever felt like committing suicide during the past year.
The EuroQol five-dimension (EQ-5D) instrument is a selfadministered questionnaire, and is the preference-based measure of health-related quality of life. ${ }^{15}$ Respondents selfclassify their health status in terms of five dimensions (mobility, self-care, ability to engage in usual activities, pain/discomfort, and anxiety/depression; scoring features three levels of severity) with three levels of severity (no problems, some problems, or extreme problems). In this study, the problems on each of the five dimensions were defined as having some or extreme problems. A unique health status is defined by combining one level from each of the five dimensions. A total of 243 health states can be defined in this way. EQ-5D health status, defined by the EQ-5D descriptive system, can be converted into a single index, the EQ-5D index, by applying a formula that essentially attaches weights to each of the levels in each dimension. ${ }^{15}$ We used the weights developed by the Korean Center for Disease Control and Prevention after considering the characteristics of Koreans; the EQ-5D index ranges from the poorest health status, -0.171 , to perfect health, $+1 .^{16}$

\section{Laboratory and anthropometric measurements}

Blood samples were collected from antecubital veins, after at least $12 \mathrm{hr}$ of fasting, processed, immediately refrigerated, and transported in the cold to the Central Testing Institute in Seoul, Korea. All blood samples were analyzed within $24 \mathrm{hr}$ after arrival. Fasting plasma glucose, triglyceride, and HDL-C levels were measured using an autoanalyzer (Hitachi Automatic Analyzer 7600; Hitachi Ltd., Tokyo, Japan). Anthropometric measurements were performed by specially trained examiners. Height and weight were measured after an overnight fast with participants wearing lightweight gowns; waist circumference was measured using a tape in the horizontal plane around the umbilical region after exhalation. Blood pressure measurements were taken in a sitting position after a rest period of at least $5 \mathrm{~min}$. BMI was calculated as weight (in $\mathrm{kg}$ ) divided by the square of height (in $\mathrm{m}$ ).

\section{Statistical analyses}

We used the SAS PROC SURVEY module, which considers strata, clusters, and weights, to analyze the data according to a complex sampling design. All analyses were performed using the sample weightings of the KNHANES. Subject characteristics by obesity subtype were explored using an analysis of variance for continuous variables and the chisquared test for dichotomous variables, with Bonferroni post hoc test. The data are expressed as means \pm standard errors, or
FIG. 1. Study population: data from the 2016 Korean National Health and Nutrition Examination Survey.

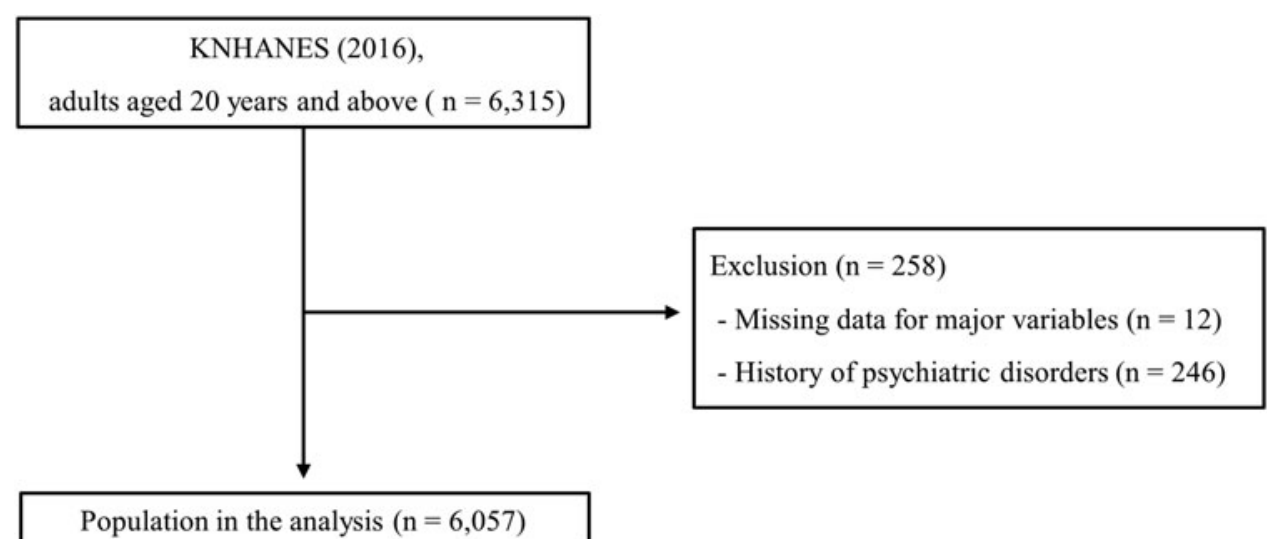

Population in the analysis $(n=6,057)$ 
percentages. The proportions of subjects with psychiatric symptoms, and scores on the various quality-of-life dimensions, by obesity subtype, were compared using the chisquared test. We considered the relationships among obesity subtypes as independent variables and the presence of psychiatric symptoms and quality-of-life problems as dependent variables. We performed a multiple logistic regression analysis after adjusting for age, sex, smoking status, alcohol consumption status, physical activity level, household income, and any history of comorbidities (dyslipidemia, diabetes mellitus, hypertension, or any infectious or inflammatory disease [sinusitis, otitis media, atopic dermatitis, or rheumatoid arthritis]). Differences in the mean EQ-5D indices by obesity subtype were evaluated via an analysis of covariance with the abovementioned variables serving as covariates. All statistical analyses were performed with the aid of SAS software (ver. 9.2; SAS Institute, Cary, NC). A $P$ value $<0.05$ was considered to indicate statistical significance.

\section{Results}

Table 1 shows the characteristics of all participants by obesity subtype. Significant differences in sex; smoking status; BMI; waist circumference; diastolic blood pressure; and the levels of fasting glucose, HbA1c, triglyceride, HDL-C, and high-sensitivity $\mathrm{C}$-reactive protein were observed among the MHNW control and MHO, MANW, and MAO groups.

The frequencies of health-related quality-of-life problems and psychiatric symptoms according to obesity subtype are shown in Table 2. Significant differences were observed in the prevalence of quality-of-life problems according to obesity subtype. The frequency of quality-of-life problems tended to increase from the MHNW to MHO, MANW, and MAO groups. The proportions of subjects reporting improper sleep durations tended to increase from the MHNW to the MAO groups ( $P$ for trend $=0.015)$. The frequency of stress in the MHO group was higher than that in the other groups $(P=0.013)$.
The unadjusted odds ratios (ORs), age- and sex-adjusted ORs, and multivariable-adjusted ORs for the five dimensions of the EQ-5D and the four psychiatric symptoms are shown in Tables 3 and 4 by obesity subtype. After adjusting for age, sex, smoking status, alcohol consumption, physical activity level, household income, and a history of comorbidities, the ORs for mobility problems in the MHO and MAO groups were 1.43 (95\% confidence interval [CI]: 1.01-2.04) and 1.94 (95\% CI: 1.52-2.47), respectively. The adjusted ORs for problems with self-care and usual activities in the MAO group were, respectively, 2.07 (95\% CI: 1.39-3.10) and 2.08 (95\% CI: 1.53-2.81). The adjusted ORs for pain and discomfort in the $\mathrm{MHO}$ and MAO groups were 1.35 (95\% CI: 1.06-1.73) and 1.39 (95\% CI: 1.14-1.69) (Table 3), respectively. In terms of psychiatric symptoms, after adjusting for the abovementioned covariates, the adjusted OR for improper sleep duration in the MAO group was 1.25 (95\% CI: 1.04 1.50) and the adjusted ORs for stress in the MHO and MAO groups were 1.27 (95\% CI: 1.05-1.54) and 1.33 (95\% CI: 1.11-1.60), respectively (Table 4).

After adjusting for the abovementioned covariates, the mean EQ-5D indices in the MHO and MAO subgroups were significantly lower than that of the MHNW control $(1.032 \pm 0.101$ and $1.023 \pm 0.101$ vs. $1.042 \pm 0.097, P=0.011$ and $<0.001$, respectively). However, the mean EQ-5D indices between MANW and MHNW subgroups did not differ (Fig. 2).

\section{Discussion}

We investigated the associations between quality of life, mental symptoms, and obesity subtype. In this study, the MHO group was positively associated with mobility and pain/discomfort problems among the five dimensions of health-related quality of life and stress, and the MAO group was positively associated with mobility, self-care, usual activities, pain/discomfort problems, and stress and improper

Table 1. Characteristics of the Study Participants by Obesity Subtypes

\begin{tabular}{|c|c|c|c|c|c|}
\hline & $M H N W$ & $\mathrm{MHO}$ & $M A N W$ & $M A O$ & $\mathrm{P}$ \\
\hline$N$ & 3138 & 897 & 784 & 1238 & - \\
\hline Age (years) & $43.9 \pm 0.4$ & $43.5 \pm 0.6$ & $59.4 \pm 0.6^{\mathrm{a}}$ & $53.2 \pm 0.6^{\mathrm{a}}$ & $<0.001$ \\
\hline Sex (male, \%) & 43.4 & $57.4^{\mathrm{a}}$ & $52.7^{\mathrm{a}}$ & $58.9^{\mathrm{a}}$ & $<0.001$ \\
\hline Current smoking $(\%)$ & 20.0 & $1.6^{\mathrm{a}}$ & $26.3^{\mathrm{a}}$ & $25.3^{\mathrm{a}}$ & 0.002 \\
\hline Heavy drinking $(\%)$ & 10.4 & $14.1^{\mathrm{a}}$ & 17.8 & $17.2^{\mathrm{a}}$ & $<0.001$ \\
\hline Physical activity (low, \%) & 50.2 & 46.0 & $61.5^{\mathrm{a}}$ & $58.4^{\mathrm{a}}$ & $<0.001$ \\
\hline Household income (low, \%) & 13.3 & 12.3 & $28.1^{\mathrm{a}}$ & $21.8^{\mathrm{a}}$ & $<0.001$ \\
\hline Body mass index $\left(\mathrm{kg} / \mathrm{m}^{2}\right)$ & $21.7 \pm 0.1$ & $27.2 \pm 0.1^{\mathrm{a}}$ & $23.1 \pm 0.1^{\mathrm{a}}$ & $28.2 \pm 0.1^{\mathrm{a}}$ & $<0.001$ \\
\hline Waist circumference $(\mathrm{cm})$ & $76.6 \pm 0.2$ & $89.7 \pm 0.3^{\mathrm{a}}$ & $83.5 \pm 0.2^{\mathrm{a}}$ & $94.4 \pm 0.3^{\mathrm{a}}$ & $<0.001$ \\
\hline Systolic blood pressure (mmHg) & $112.9 \pm 0.3$ & $118.6 \pm 0.6$ & $127.8 \pm 0.7^{\mathrm{a}}$ & $126.3 \pm 0.5^{\mathrm{a}}$ & $<0.001$ \\
\hline Diastolic blood pressure (mmHg) & $73.3 \pm 0.2$ & $77.0 \pm 0.4^{\mathrm{a}}$ & $78.0 \pm 0.5^{\mathrm{a}}$ & $81.1 \pm 0.4^{\mathrm{a}}$ & $<0.001$ \\
\hline Fasting glucose (mg/dL) & $93.3 \pm 0.3$ & $97.9 \pm 1.3^{\mathrm{a}}$ & $113.6 \pm 1.4^{\mathrm{a}}$ & $114.4 \pm 1.0^{\mathrm{a}}$ & $<0.001$ \\
\hline Total cholesterol (mg/dL) & $190.9 \pm 0.8$ & $199.5 \pm 1.4^{\mathrm{a}}$ & $192.5 \pm 2.0$ & $195.4 \pm 1.4$ & $<0.001$ \\
\hline LDL-cholesterol (mg/dL) & $117.9 \pm 3.2$ & $129.8 \pm 3.5$ & $108.8 \pm 2.5^{\mathrm{a}}$ & $118.2 \pm 1.8$ & $<0.001$ \\
\hline Triglycerides $(\mathrm{mg} / \mathrm{dL})$ & $106.8 \pm 2.4$ & $126.4 \pm 2.8^{\mathrm{a}}$ & $225.8 \pm 13.7^{\mathrm{a}}$ & $212.9 \pm 4.4^{\mathrm{a}}$ & $<0.001$ \\
\hline HDL-cholesterol (mg/dL) & $55.5 \pm 0.3$ & $51.0 \pm 0.4^{\mathrm{a}}$ & $44.0 \pm 0.5^{\mathrm{a}}$ & $43.6 \pm 0.3^{\mathrm{a}}$ & $<0.001$ \\
\hline $\mathrm{HbA}_{1} \mathrm{C}(\%)$ & $5.4 \pm 0.01$ & $5.6 \pm 0.03^{\mathrm{a}}$ & $6.0 \pm 0.03^{\mathrm{a}}$ & $6.1 \pm 0.03^{\mathrm{a}}$ & $<0.001$ \\
\hline hs-CRP (mg/L) & $1.0 \pm 0.04$ & $1.4 \pm 0.10^{\mathrm{a}}$ & $1.5 \pm 0.10^{\mathrm{a}}$ & $1.7 \pm 0.10^{\mathrm{a}}$ & $<0.001$ \\
\hline
\end{tabular}

The values are percentages for categorical variables and means \pm standard errors for continuous variables.

${ }^{a}$ Significantly different from MHNW group $(P$ value $<0.05)$.

HDL, high-density lipoprotein; hs-CRP, high-sensitivity C-reactive protein; LDL, low-density lipoprotein; MANW, metabolically abnormal but of normal weight; MAO, metabolically abnormal and obese; MHNW, metabolically healthy normal weight; MHO, metabolically healthy but obese. 
Table 2. The Frequencies of Quality-of-Life Problems and the EuroQol Five-Dimension Indices by Obesity SubTypes

\begin{tabular}{lrrrrrr}
\hline & MHNW & MHO & MANW & MAO & $\mathrm{P}$ & P for trend \\
\hline Quality of life problems & & & & & & \\
$\quad$ Mobility & 7.5 & 8.7 & 17.2 & 19.7 & $<0.001$ & $<0.001$ \\
$\quad$ Self-care & 1.7 & 1.8 & 5.8 & 5.5 & $<0.001$ & $<0.001$ \\
Usual activities & 3.9 & 3.4 & 10.9 & 11.7 & $<0.001$ & $<0.001$ \\
Pain/discomfort & 15.9 & 18.6 & 24.2 & 24.4 & $<0.001$ & $<0.001$ \\
$\quad$ Anxiety/depression & 8.4 & 7.2 & 12.0 & 10.5 & 0.005 & 0.013 \\
Psychiatric symptoms & & & & & & \\
$\quad$ Improper sleep & 36.2 & 37.8 & 37.4 & 41.3 & 0.063 & 0.015 \\
$\quad$ Stress & 27.6 & 31.1 & 22.9 & 29.0 & 0.013 & $>0.999$ \\
Depressed mood & 19.9 & 19.9 & 22.7 & 19.9 & 0.549 & 0.609 \\
$\quad$ Suicidal ideation & 1.5 & 1.6 & 1.8 & 1.9 & 0.842 & 0.392 \\
\hline
\end{tabular}

All values are expressed as percentages.

sleep. The EQ-5D indices, an assessment of overall quality of life, were significantly lower in both $\mathrm{MHO}$ and MAO groups than in MHNW control.

Obesity is not only associated with many metabolic and chronic diseases, but it is also related to one's mental health and quality of life. ${ }^{1,2,8,17,18}$ In this study, we found that obesity (both MHO and MAO groups) was associated with decreased quality of life and increased psychiatric symptoms, consistent with the findings of previous studies. ${ }^{8,18}$ However, this study found no significant worsening of the quality of life or psychiatric status of the MANW group. Several studies reported relationships between MetS and quality of life, ${ }^{10}$ but others did not. ${ }^{19,20}$ In a crosssectional study, an association between MetS and poor quality of life was evident only in the physical health domain of the SF-36 index; the association disappeared after adjustment for BMI, and was thus explained by the BMI increase alone. ${ }^{10}$ Another cross-sectional study of the health-related quality of life of Korean adults found no significant association between MetS and quality of life after adjusting for age, sex, and smoking status, ${ }^{20}$ suggesting that the extent of metabolic disturbance may not be linearly related to such mental problems and quality of life.

Table 3. Associations Between Quality-of-Life Problems and Obesity Subtypes

\begin{tabular}{|c|c|c|c|c|c|c|}
\hline & \multicolumn{2}{|c|}{ Crude } & \multicolumn{2}{|c|}{ Model 1} & \multicolumn{2}{|c|}{ Model 2} \\
\hline & OR $(95 \% C I)$ & $\mathrm{P}$ & OR $(95 \% C I)$ & $\mathrm{P}$ & OR $(95 \% C I)$ & $\mathrm{P}$ \\
\hline \multicolumn{7}{|l|}{ Mobility } \\
\hline MHNW & 1 & & 1 & & 1 & \\
\hline MHO & $1.19(0.88-1.59)$ & 0.253 & $1.33(0.97-1.84)$ & 0.080 & $1.43(1.01-2.04)$ & 0.045 \\
\hline MANW & $2.58(2.09-3.20)$ & $<0.001$ & $1.13(0.91-1.40)$ & 0.281 & $1.00(0.78-1.29)$ & 0.989 \\
\hline MAO & $3.04(2.49-3.71)$ & $<0.001$ & $2.01(1.60-2.52)$ & $<0.001$ & $1.94(1.52-2.47)$ & $<0.001$ \\
\hline \multicolumn{7}{|l|}{ Self-care } \\
\hline MHNW & 1 & & 1 & & 1 & \\
\hline MHO & $1.03(0.57-1.88)$ & 0.913 & $1.15(0.63-2.13)$ & 0.644 & $1.22(0.65-2.30)$ & 0.537 \\
\hline MANW & $3.45(2.30-5.18)$ & $<0.001$ & $1.44(0.95-2.17)$ & 0.085 & 1.39 (0.86-2.27) & 0.178 \\
\hline MAO & $3.25(2.23-4.74)$ & $<0.001$ & $1.96(1.32-2.91)$ & 0.001 & $2.07(1.39-3.10)$ & 0.001 \\
\hline \multicolumn{7}{|c|}{ Usual activities } \\
\hline MHNW & 1 & & 1 & & 1 & \\
\hline MHO & $0.85(0.54-1.34)$ & 0.476 & $0.91(0.57-1.46)$ & 0.697 & $0.88(0.55-1.42)$ & 0.602 \\
\hline MANW & $2.98(2.22-4.01)$ & $<0.001$ & $1.39(1.03-1.87)$ & 0.031 & $1.25(0.86-1.81)$ & 0.234 \\
\hline MAO & $3.23(2.49-4.18)$ & $<0.001$ & $2.12(1.59-2.83)$ & $<0.001$ & $2.08(1.53-2.81)$ & $<0.001$ \\
\hline \multicolumn{7}{|c|}{ Pain/discomfort } \\
\hline MHNW & 1 & & 1 & & 1 & \\
\hline MHO & $1.20(0.95-1.53)$ & 0.127 & $1.32(1.03-1.68)$ & 0.027 & $1.35(1.06-1.73)$ & 0.017 \\
\hline MANW & $1.69(1.41-2.03)$ & $<0.001$ & $1.19(0.98-1.46)$ & 0.085 & $1.11(0.88-1.40)$ & 0.387 \\
\hline MAO & $1.71(1.43-2.04)$ & $<0.001$ & $1.46(1.21-1.76)$ & $<0.001$ & $1.39(1.14-1.69)$ & 0.001 \\
\hline \multicolumn{7}{|c|}{ Anxiety/depression } \\
\hline MHNW & 1 & & 1 & & 1 & \\
\hline MHO & $0.85(0.62-1.18)$ & 0.341 & $0.93(0.67-1.28)$ & 0.639 & $0.94(0.67-1.32)$ & 0.714 \\
\hline MANW & $1.49(1.14-1.95)$ & 0.004 & $1.34(1.01-1.78)$ & 0.046 & $1.21(0.88-1.68)$ & 0.244 \\
\hline MAO & $1.29(0.99-1.67)$ & 0.060 & $1.27(0.97-1.66)$ & 0.088 & $1.17(0.89-1.54)$ & 0.268 \\
\hline
\end{tabular}

Model 1: adjusted for age and sex; Model 2: adjusted for age, sex, alcohol consumption, smoking, exercise, income, a history of comorbidities.

CI, confidence interval; OR, odds ratio. 
Table 4. Associations Between Psychiatric Symptoms and Obesity Subtypes

\begin{tabular}{|c|c|c|c|c|c|c|}
\hline & \multicolumn{2}{|c|}{ Crude } & \multicolumn{2}{|c|}{ Model 1} & \multicolumn{2}{|c|}{ Model 2} \\
\hline & OR $(95 \% C I)$ & $\mathrm{P}$ & OR $(95 \% C I)$ & $\mathrm{P}$ & OR $(95 \% C I)$ & $\mathrm{P}$ \\
\hline \multicolumn{7}{|c|}{ Improper sleep } \\
\hline MHNW & 1 & & 1 & & 1 & \\
\hline MHO & $1.07(0.90-1.29)$ & 0.440 & $1.08(0.90-1.29)$ & 0.421 & $1.11(0.93-1.32)$ & 0.244 \\
\hline MANW & $1.05(0.87-1.27)$ & 0.592 & $1.04(0.85-1.27)$ & 0.704 & $1.05(0.84-1.30)$ & 0.664 \\
\hline MAO & $1.24(1.05-1.46)$ & 0.010 & $1.24(1.04-1.47)$ & 0.016 & $1.25(1.05-1.50)$ & 0.015 \\
\hline \multicolumn{7}{|l|}{ Stress } \\
\hline MHNW & 1 & & 1 & & 1 & \\
\hline MHO & $1.18(0.98-1.42)$ & 0.081 & $1.21(1.00-1.46)$ & 0.049 & $1.27(1.05-1.54)$ & 0.017 \\
\hline MANW & $0.78(0.63-0.96)$ & 0.021 & $1.12(0.89-1.40)$ & 0.324 & $1.01(0.80-1.28)$ & 0.910 \\
\hline MAO & $1.07(0.90-1.26)$ & 0.441 & $1.36(1.14-1.63)$ & 0.001 & $1.33(1.11-1.60)$ & 0.003 \\
\hline \multicolumn{7}{|c|}{ Depressed mood } \\
\hline MHNW & 1 & & 1 & & 1 & \\
\hline MHO & $1.00(0.80-1.25)$ & 0.998 & $1.10(0.87-1.38)$ & 0.435 & $1.08(0.85-1.38)$ & 0.535 \\
\hline MANW & $1.18(0.95-1.47)$ & 0.136 & $1.39(1.11-1.73)$ & 0.004 & $1.16(0.90-1.49)$ & 0.250 \\
\hline MAO & $1.00(0.83-1.22)$ & 0.973 & $1.18(0.96-1.45)$ & 0.112 & $1.04(0.84-1.29)$ & 0.721 \\
\hline \multicolumn{7}{|c|}{ Suicidal ideation } \\
\hline MHNW & 1 & & 1 & & 1 & \\
\hline MHO & $1.05(0.56-1.97)$ & 0.868 & $1.10(0.59-2.06)$ & 0.753 & $1.09(0.58-2.06)$ & 0.785 \\
\hline MANW & $1.21(0.64-2.29)$ & 0.553 & $0.82(0.43-1.58)$ & 0.556 & $0.55(0.28-1.06)$ & 0.072 \\
\hline MAO & $1.27(0.69-2.32)$ & 0.437 & $1.02(0.53-1.94)$ & 0.959 & $0.70(0.36-1.36)$ & 0.288 \\
\hline
\end{tabular}

Model 1: adjusted for age and sex; Model 2: adjusted for age, sex, alcohol consumption, smoking, exercise, income, a history of comorbidities.

The association between obesity and mental health may be bidirectional. There are some factors related to both obesity and psychiatric disorders, such as diet, exercise, genetic trait, medication, and neurotransmitter imbalance. These factors are hypothesized to explain this relationship. For example, dysregulated cortisol can be assumed to be a player in both mood and weight regulation, because the changes in the hypothalamic/pituitary/adrenal axis seen in depression are similar to those seen in Cushing syndrome and endocrine illness characterized by weight gain. ${ }^{6}$ In addition, patients with attention-deficit/hyperactivity dis-

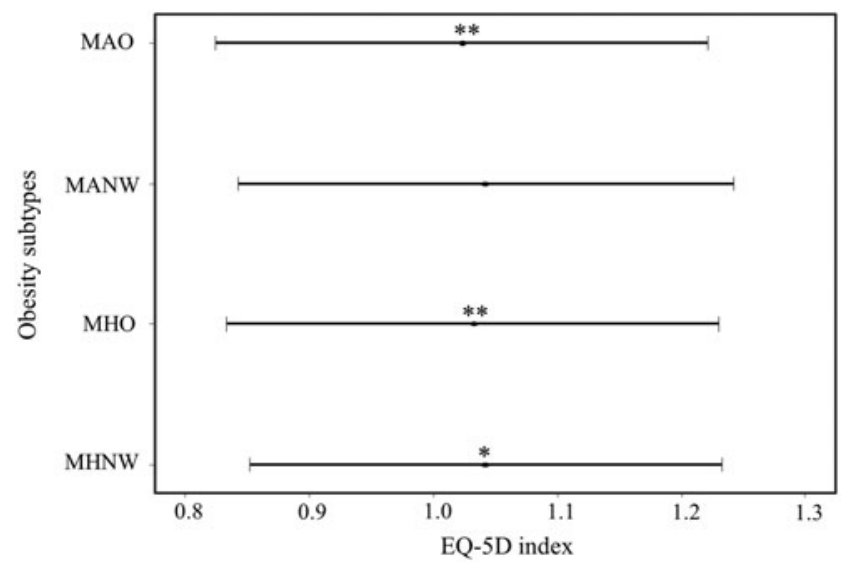

FIG. 2. EQ-5D index according to obesity subtype. Data are expressed as means \pm standard errors. $*$ Reference, $* * P$ value $<0.05$. EQ-5D, EuroQol five-dimension; MANW, metabolically abnormal but of normal weight; MAO, metabolically abnormal and obese; MHNW, metabolically healthy normal weight. order, post-traumatic stress disorder, or schizophrenia might have difficulty in having organized meals and the proper amount of physical activity, which can lead to increased risk of obesity. These psychiatric disorders are also associated with hormones such as dopamine and serotonin, which are also related to obesity. ${ }^{6}$ Furthermore, obesity is not just related to mental problems but is also linked to disability and comorbidities and can have a negative effect on the quality of life. MetS is a risk factor of hypertension, type 2 diabetes mellitus, dyslipidemia and atherosclerotic cardiovascular diseases, and concomitant obesity is a major driver of MetS. ${ }^{21}$ There have been many studies on the association of these chronic diseases and quality of life, and it has been found that there is a negative effect on the quality of life. ${ }^{22,23}$ Considering these points, it can be assumed that when obesity is accompanied by metabolic disturbance, its link with mental health problems and poor quality of life will be strengthened.

The strength of our study is that this is the first nationally representative population-based work exploring quality of life and mental health status by obesity subtype in South Korea. However, several limitations are apparent. First, the study was cross sectional. Second, as some data (including psychiatric symptoms) were self-reported, objectivity might be lacking.

\section{Conclusion}

Obesity is associated with mental health problems and the decreased quality of life, and obesity with metabolic abnormalities is also related to poor quality of life and mental symptoms, suggesting that obesity in those with or without metabolic disturbances is associated with mental health problems and decreased quality of life. 


\section{Authors' Contributions}

Conceptualization: S.-W.S.; data curation: H.-N.K. and S.-W.S.; formal analysis: S.-R.K., H.-N.K., and S.-W.S.; writing — original draft preparation: S.-R.K.; writing - review and editing: S.-R.K., H.-N.K., and S.-W.S.; supervision: H.-N.K. and S.-W.S.

\section{Acknowledgments}

Statistical consultation was supported by the Department of Biostatistics of the Catholic Research Coordinating Center.

\section{Ethics Approval}

All protocols of the Korean National Health and Nutrition Examination Survey have been approved by the Institutional Review Board of the Korean Center for Disease Control and Prevention, and all participants provided written informed consent at baseline. This study was approved by the Institutional Review Board of the Catholic University of Korea (IRB approval number: VC18ZESI0158).

\section{Consent for Publication}

It was obtained from all the participants included.

\section{Availability of Data and Material}

The data are available from Korean Centers for Disease Control and Prevention, Korea National Health and Nutrition Examination Survey (http://knhanes.cdc.go.kr) on request.

\section{Author Disclosure Statement}

No conflicting financial interests exist.

\section{Funding Information}

This research did not receive any specific grant from funding agencies in the public, commercial, or not-for-profit sectors.

\section{References}

1. Williams EP, Mesidor M, Winters K, et al. Overweight and obesity: Prevalence, consequences, and causes of a growing public health problem. Curr Obes Rep 2015;4:363-370.

2. Chu DT, Minh Nguyet NT, Dinh TC, et al. An update on physical health and economic consequences of overweight and obesity. Diabetes Metab Syndr 2018;12:1095-1100.

3. Cappuccio FP, Miller MA. Sleep and cardio-metabolic disease. Curr Cardiol Rep 2017;19:110-110.

4. Martin DJ, Ul-Haq Z, Nicholl BI, et al. Cardiometabolic disease and features of depression and bipolar disorder: Population-based, cross-sectional study. $\mathrm{Br} J$ Psychiatry 2016;208:343-351.

5. Chwastiak L, Fortney J. Learning to integrate cardiometabolic care in serious mental illness. Am J Psychiatry 2017; 174:199-201.

6. Avila C, Holloway AC, Hahn MK, et al. An overview of links between obesity and mental health. Curr Obes Rep 2015;4:303-310.

7. Karelis AD, St-Pierre DH, Conus F, et al. Metabolic and body composition factors in subgroups of obesity: What do we know? J Clin Endocrinol Metab 2004;89:2569-2575.
8. Donini LM, Merola G, Poggiogalle E, et al. Disability, physical inactivity, and impaired health-related quality of life are not different in metabolically healthy vs. unhealthy obese subjects. Nutrients 2016;8.

9. Lopez-Garcia E, Guallar-Castillon P, Garcia-Esquinas E, et al. Metabolically healthy obesity and health-related quality of life: A prospective cohort study. Clin Nutr 2017;36:853-860.

10. Tsai AG, Wadden TA, Sarwer DB, et al. Metabolic syndrome and health-related quality of life in obese individuals seeking weight reduction. Obesity (Silver Spring) 2008;16:59-63.

11. Phillips CM, Perry IJ. Depressive symptoms, anxiety and well-being among metabolic health obese subtypes. Psychoneuroendocrinology 2015;62:47-53.

12. Wen CP, David Cheng TY, Tsai SP, et al. Are Asians at greater mortality risks for being overweight than Caucasians? Redefining obesity for Asians. Public Health Nutr 2009;12:497-506.

13. Grundy SM, Cleeman JI, Daniels SR, et al. Diagnosis and management of the metabolic syndrome: An American Heart Association/National Heart, Lung, and Blood Institute Scientific Statement. Circulation 2005;112:2735-2752.

14. Lee SY, Park HS, Kim DJ, et al. Appropriate waist circumference cutoff points for central obesity in Korean adults. Diabetes Res Clin Pract 2007;75:72-80.

15. Herdman M, Gudex C, Lloyd A, et al. Development and preliminary testing of the new five-level version of EQ-5D (EQ-5D-5L). Qual Life Res 2011;20:1727-1736.

16. Lee YK, Nam HS, Chuang LH, et al. South Korean time tradeoff values for EQ-5D health states: Modeling with observed values for 101 health states. Value Health 2009;12:1187-1193.

17. Heymsfield SB, Wadden TA. Mechanisms, pathophysiology, and management of obesity. N Engl J Med 2017; 376:254-266.

18. Kolotkin RL, Andersen JR. A systematic review of reviews: Exploring the relationship between obesity, weight loss and health-related quality of life. Clin Obes 2017;7:273-289.

19. Saboya PP, Bodanese LC, Zimmermann PR, et al. Metabolic syndrome and quality of life: A systematic review. Rev Lat Am Enfermagem 2016;24:e2848.

20. Lee YJ, Woo SY, Ahn JH, et al. Health-related quality of life in adults with metabolic syndrome: The Korea national health and nutrition examination survey, 2007-2008. Ann Nutr Metab 2012;61:275-280.

21. Grundy SM. Metabolic syndrome update. Trends Cardiovasc Med 2016;26:364-373.

22. Han JH, Park HS, Shin CI, et al. Metabolic syndrome and quality of life (QOL) using generalised and obesity-specific QOL scales. Int J Clin Pract 2009;63:735-741.

23. Park SS, Yoon YS, Oh SW. Health-related quality of life in metabolic syndrome: The Korea National Health and $\mathrm{Nu}-$ trition Examination Survey 2005. Diabetes Res Clin Pract 2011;91:381-388.

Address correspondence to: Sang-Wook Song, $M D, P h D$ Department of Family Medicine St. Vincent's Hospital College of Medicine The Catholic University of Korea Jungbu-daero 93, Paldal-gu Suwon-si 16247 Gyeonggi-do Republic of Korea

E-mail:sswkoj@unitel.co.kr 